\title{
Metabolomic response of osteosarcoma cells to nanographene oxide-mediated hyperthermia
}

\author{
Mónica Cicuéndez ${ }^{\mathrm{a}, \mathrm{b}}$, , Joana Flores ${ }^{\mathrm{a}}$, Helena Oliveira ${ }^{\mathrm{a}, \mathrm{c}}$, M. Teresa Portolés ${ }^{\mathrm{d}}$, María Vallet-Regí ${ }^{e, \mathrm{f}}$, \\ Mercedes Vila ${ }^{\mathrm{b}, 1}$, Iola F. Duarte ${ }^{\mathrm{a}, * *}, 1$ \\ ${ }^{a}$ CICECO-Aveiro Institute of Materials, Department of Chemistry, University of Aveiro (UA), Aveiro, Portugal \\ ${ }^{\mathrm{b}}$ NRG-TEMA, Department of Mechanical Engineering, University of Aveiro (UA), Portugal \\ c CESAM, Department of Biology, University of Aveiro (UA), Aveiro, Portugal \\ d Department of Biochemistry and Molecular Biology I, Faculty of Chemistry, Universidad Complutense de Madrid, Instituto de Investigación Sanitaria del Hospital Clínico San Carlos (IdISSC), \\ Ciudad Universitaria $s / n, 28040$ Madrid, Spain \\ ${ }^{\mathrm{e}}$ Department of Inorganic and Bioinorganic Chemistry, Faculty of Pharmacy, Universidad Complutense de Madrid, Instituto de Investigación Sanitaria Hospital 12 de Octubre i+ 12, Ciudad \\ Universitaria s/n, 28040 Madrid, Spain \\ ${ }^{\mathrm{f}}$ Networking Research Center on Bioengineering, Biomaterials and Nanomedicine, CIBER-BBN, Spain
}

\section{ART ICLE INFO}

\section{Keywords:}

Pegylated nanographene oxide (nGO) sheets Hyperthermia

Cancer

Saos-2 osteoblasts

HRMAS ${ }^{1} \mathrm{H}$ NMR

Metabolomics

Cell metabolism

\begin{abstract}
A B S T R A C T
Nanographene oxide (nGO)-mediated hyperthermia has been increasingly investigated as a localized, minimally invasive anticancer therapeutic approach. Near InfraRed (NIR) light irradiation for inducing hyperthermia is particularly attractive, because biological systems mostly lack chromophores that absorb in this spectral window, facilitating the selective heating and destruction of cells which have internalized the NIR absorbing-nanomaterials. However, little is known about biological effects accompanying nGO-mediated hyperthermia at cellular and molecular levels. In this work, well-characterized pegylated nGO sheets with a hydrodynamic size of $300 \mathrm{~nm}$ were incubated with human Saos-2 osteosarcoma cells for $24 \mathrm{~h}$ and their internalization verified by flow cytometry and confocal microscopy. No effect on cell viability was observed after nGO uptake by Saos- 2 cells. However, a proliferation delay was observed due to the presence of nGO sheets in the cytoplasm. ${ }^{1} \mathrm{H}$ NMR metabolomics was employed to screen for changes in the metabolic profile of cells, as this could help to improve understanding of cellular responses to nanomaterials and provide new endpoint markers of effect. Cells internalizing nGO sheets showed noticeable changes in several metabolites compared to control cells, including decreased levels of several amino acids, taurine and creatine and increased levels of phosphocholine and uridine/adenosine nucleotides. After NIR irradiation, cells showed decreases in glutamate and uridine nucleotides, together with increases in glycerophosphocholine and adenosine monophosphate. Overall, this study has shown that the cellular metabolome sensitively responded to nGO exposure and nGO-mediated hyperthermia and that NMR metabolomics is a powerful tool to investigate treatment responses.
\end{abstract}

\section{Introduction}

The development of alternative and/or complementary therapeutic strategies that can efficiently eliminate tumor cells without causing major deleterious side effects is a crucially important goal in the fight against cancer [1-6]. Tumor destruction through nanomaterial-mediated hyperthermia has been increasingly investigated as a minimally invasive alternative to surgery, as well as to treat tumors embedded in vital regions where surgical resection is not feasible $[7,8]$. In this type of therapy, an energy-absorbing nanosystem localized within tumor tissues absorbs energy provided

\footnotetext{
* Correspondence to: Iola F. Duarte, CICECO-Aveiro Institute of Materials, Department of Chemistry, University of Aveiro (UA), Aveiro, Portugal.

* Corresponding author.

Email addresses: mcicuendez@ua.pt (M. Cicuéndez); ioladuarte@ua.pt (I.F. Duarte)

1 These authors contributed equally to this work.
} 
by an external source and converts it into heat, thereby inducing localized thermal destruction (above $40^{\circ} \mathrm{C}$ ) without affecting the surrounding tissues, which have not taken up the nanomaterial [9-11]. The use of Near Infrared (NIR) light in the 700-1100 nm range for inducing tumor hyperthermia is particularly attractive, because biological systems mostly lack chromophores that absorb in this spectral window, facilitating the selective heating and destruction of cells which have internalized the NIR absorbing-nanomaterials [12-14].

Several nanomaterials are being developed for use in nanotechnology-based hyperthermia methods [15-20]. Nanosized graphene, particularly graphene oxide (nGO), represents one of the most thrilling candidates for this application, especially due to its strong NIR optical absorption ability [21-23]. Moreover, nGO has other fascinating properties which make it exquisitely suitable for nanomedicine applications, such as excellent aqueous processability, amphiphilicity, Surface Enhanced Raman Scattering (SERS) property, and fluorescence quenching ability [24].

Nanographene oxide-mediated hyperthermia has been studied in vitro [25-27] and in vivo [28,29]. It is known that depending on the laser fluency, different temperature increments and various thermodynamic and thermo-biological responses can occur in the tissue medium [30]. However, there is still limited understanding of the biological effects accompanying nGO-mediated hyperthermia at the cellular and molecular levels. For instance, to our knowledge, no data concerning the metabolic changes produced in tumor cells by nGO-mediated hyperthermia have been described so far. The cellular metabolome (inventory of small molecules acting as substrates/products of enzyme-mediated reactions) closely reflects biochemical activity and functional status, being currently recognized as a key player in the cellular response to external stimuli such as nanomaterials $[31,32]$. Hence, the ability to comprehensively describe multiple metabolite changes (in a single analytical run) is expected to provide improved understanding of biological processes, compared to conventional single endpoint readouts. ${ }^{1} \mathrm{H}$ NMR is exquisitely useful in metabolomics due to its unparalleled analytical reproducibility and ability to provide unequivocal structural and quantitative information on a wide range of metabolites. Indeed, all proton-containing small molecules at submillimolar concentrations may potentially be detected in complex biological samples (such as cells, tissues or biofluids), distinguished based on their chemical shifts and multiplicities, and quantified based on signal areas [33].

In the present study, well-characterized pegylated nGO sheets (size of $300 \mathrm{~nm}$ ) were incubated with human Saos-2 osteosarcoma cells for $24 \mathrm{~h}$ and their internalization verified, along with the impact on cell viability and proliferation. Saos-2 osteosarcoma cells have been selected as a human tumor cell model due to their highly conserved properties. Indeed, this cell line has been previously used in a large variety of in vitro studies with nanomaterials based on its osteoblastic features, such as expression of collagen type I and type III, production of mineralized matrix, high alkaline phosphatase levels, PTH receptors coupled to adenylate cyclase and presence of osteonectin (OC), bone sialoprotein (BSP) and procollagen-I [34-36]. ${ }^{1} \mathrm{H}$ NMR analysis of lysed cell pellets was employed to assess the changes in the cellular metabolome induced by nGO uptake and subsequent laser irradiation $\left(1.5 \mathrm{~W} / \mathrm{cm}^{2}\right.$ power, $\left.5 \mathrm{~min}\right)$. This approach is expected to help improving current understanding of cellular responses to nGO-mediated hyperthermia and to provide potential endpoint markers of effect.

\section{Materials and methods}

\subsection{Synthesis and characterization of pegylated $n G O$ sheets}

Pegylated nGO sheets have been obtained from exfoliation of high-purity graphite in an acidic medium by a modified Hummers method, as previously reported [37]. The resulting nGO suspension was dialyzed until pH7, and activated with chloroacetic acid $\left(\mathrm{Cl}-\mathrm{CH}_{2}-\mathrm{COOH}\right)$ under strongly basic conditions $(\mathrm{NaOH})$ to promote $-\mathrm{COOH}$ groups at the surface. Then, it was functionalized by covalent bonding (diimide activation by adding EDAC:1-Ethyl-3-(3-dimethylaminopropyl) carbodiimide hydrochloride) with the non-toxic and non-immunogenic polymer Poly(Ethylene Glycolamine)(PEG) to improve coloidal stability and minimize immunogenicity. Finally, nGO sheets linked to 1-arm PEG were marked with the amine reactive dye fluorescein isothiocyanate (FITC) covalently bonded to the PEG. The resulting samples were analyzed by Atomic Force Microscopy AFM multimode Nanoscope III A (Bruker). Dynamic light scattering (DLS) measurements were also performed in pH5 solutions in a Zetasizer Nano series instrument equipped with a $633 \mathrm{~nm}$ "red" laser from Malvern Instruments, with reproducibility being verified by collection and comparison of sequential measurements. $Z$-average sizes of three sequential measurements were collected at room temperature (RT) and analyzed. X-ray Photoelectron Spectroscopy (XPS) spectra were acquired in an U1tra High Vacuum (UHV) system with a base pressure of $2 \times 10^{-10}$ mbar. nGO-PEG powder was dispersed in $\mathrm{MQ} \mathrm{H}_{2} \mathrm{O}$ and drop coated on a $\mathrm{Si}$ wafer. The system is equipped with a hemispherical electron energy analyzer (SPECS Phoibos 150), a delay-line detector and a monochromatic AlK $\alpha(1486.74 \mathrm{eV}) \mathrm{X}$-ray source. High resolution spectra were recorded at normal emission take-off angle and with a pass-energy of $20 \mathrm{eV}$, which provides an overall instrumental peak broadening of $0.5 \mathrm{eV}$. The XPS spectra were calibrated in binding energy by referencing to the first component of the $\mathrm{C} 1 \mathrm{~s}$ spectrum to $284.8 \mathrm{eV}$.

\subsection{Cell culture for nGO uptake and cell proliferation studies}

Human Saos-2 osteoblasts were seeded at a density of $10^{5}$ cells $/ \mathrm{mL}$ in DMEM culture medium supplemented with $10 \%$ FBS, 1 mML-glutamine, penicillin, streptomycin, under a $5 \% \mathrm{CO}_{2}$ atmosphere and at $37^{\circ} \mathrm{C}$ for $24 \mathrm{~h}$. After this time, $75 \mu \mathrm{g} / \mathrm{mL}$ of $\mathrm{nGO}$ material were added to the medium and the cells were cultured for $24 \mathrm{~h}$ in contact with nGO suspension. This concentration was chosen based on previous results whereby Saos-2 cells were found to rapidly internalize nGO and to maintain high cell viability at this exposure level [38]. Then, the attached cells were harvested with $0.25 \%$ trypsin-EDTA and counted with a Neubauer hemocytometer, using trypan blue for assessing viability. For assessing nGO cellular uptake, the fluorescence of nGO-FITC was excited at $488 \mathrm{~nm}$ and measured with a 530/30band pass filter in an Attune ${ }^{\circledR}$ Acoustic Focusing Cytometer (ThermoFisher Scientific, Waltham, MA). The conditions for data acquisition and flow cytometric analysis were established using negative and positive controls and these conditions were maintained during all the experiments. Each experiment was carried out three times and single representative experiments are displayed. For statistical significance, at least $10^{5}$ cells were analyzed in each sample and the mean fluorescence emitted by these single cells was estimated from the mean fluorescence intensity (MFI) of FITC using the FlowJo software (Tree Star Inc., Ashland, OR, USA). 


\subsection{Confocal microscopy studies}

Cells were seeded on glass coverslips and cultured in the presence of pegylated nGO material for $24 \mathrm{~h}$, fixed with $3.7 \%$ paraformaldehyde in PBS, permeabilizated with $0.1 \%$ Triton X-100 and preincubated with PBS containing $1 \%$ BSA. Then, cells were incubated for 20 min with rhodamine-phalloidin (1:40), stained with 40-6-diamidino-20-phenylindole (DAPI, $3 \times 10^{-6} \mathrm{M}$ in PBS) and examined using a LeicaSP2 Confocal Laser Scanning Microscope. Rhodamine fluorescence was excited at $540 \mathrm{~nm}$ and measured at $565 \mathrm{~nm}$. DAPI fluorescence was excited at $405 \mathrm{~nm}$ and measured at $420-480 \mathrm{~nm}$.

\subsection{NIR laser irradiation}

NIR radiation was provided by a high-power $(30 \mathrm{~W})$ diodelaser (LASING S.A.) emitting in $808 \mathrm{~nm}$, giving a circular irradiation area of diameter $3 \mathrm{~cm}$ (fluency: $4 \mathrm{~J} \mathrm{~cm}^{-2}$ ). The module allows irradiation of culture plates in a sterile environment. Irradiation was performed after nGO-cell uptake which, based on previous results, was considered complete after $24 \mathrm{~h}$ of Saos-2 exposure to nGO solution. Laser irradiation was performed at $1.5 \mathrm{~W} / \mathrm{cm}^{2}$ power during $5 \mathrm{~min}$, based on previously reported results [30]. Immediately ( $3 \mathrm{~min})$ after laser irradiation, cells were harvested using $0.25 \%$ trypsin-EDTA, washed with deuterated PBS (pH7.4), centrifuged $\left(1000 \mathrm{~g}, 6 \mathrm{~min}, 4^{\circ} \mathrm{C}\right.$ ), re-suspended in $40 \mu \mathrm{L} \mathrm{PBS} /$ $\mathrm{D}_{2} \mathrm{O}$, and stored at $-80^{\circ} \mathrm{C}$ until Nuclear Magnetic Resonance (NMR) analysis.

\subsection{NMR analysis}

Thawed cells were mechanically lysed by a three-fold cycle of nitrogen freezing and sonication [39], and $50 \mu \mathrm{L}$ of each sample were transferred into a High Resolution Magic Angle Spinning (HR-MAS) rotor. NMR spectra were acquired on a Bruker Avance DRX-500 spectrometer operating at $500.13 \mathrm{MHz}$ for ${ }^{1} \mathrm{H}$ observation, at $277 \mathrm{~K}$, using a $4 \mathrm{~mm}$ HRMAS probe, in which the rotor containing the sample was spun at $4 \mathrm{kHz}$. To attenuate broad signals of macromolecules and improve the detection of small metabolites, the T2-edited Carr-Purcell-Meiboom-Gill spin-echo pulse sequence with water presaturation ("cpmgpr1d" in Bruker library) was employed, using a total spin echo time of $60 \mathrm{~ms}(n=150, \tau=200 \mu \mathrm{s})$. Each 1D spectrum was acquired with 2048 transients, $32 \mathrm{k}$ data points, a spectral width of $6510.42 \mathrm{~Hz}$, a relaxation delay of $2 \mathrm{~s}$, and an acquisition time of $2.5 \mathrm{~s}$. Spectra were processed with a line broadening of $0.3 \mathrm{~Hz}$ and zero-filling factor of 2 , manually phased and baseline corrected. Chemical shifts were referenced internally to the alanine signal at $\delta 1.48 \mathrm{ppm}$. Spectral assignment was based on 2D total correlation spectroscopy (TOCSY) and heteronuclear single quantum coherence (HSQC) spectra and consultation of spectral databases, such as the Bruker Biorefcode database and the human metabolome HMDB database [40].

\subsection{Multivariate analysis and integration of NMR data}

Data matrices of total area-normalized spectra were built in Amix-viewer (version 3.9.14, Bruker Biospin, Rheinstetten) and multivariate analysis (MVA) was performed in SIMCA-P11.5 (Umetrics, Sweden). Principal component analysis (PCA) was followed by partial least square discriminant analysis (PLS-DA), whereby a 7-fold internal cross-validation was applied to assess the explained variance $\left(\mathrm{R}^{2}\right)$ and predictive power $\left(Q^{2}\right)$. The corresponding load- ings were obtained by multiplying the loading weight $(\mathrm{w})$ by the standard deviation of each variable, and were color-coded according to variable importance to the projection (VIP).

To assess the magnitude of metabolite variations, selected spectral peaks were integrated in Amix-viewer, and normalized by the total area of each spectrum (excluding the suppressed water signal). For each metabolite, the magnitude of variation in nGO-treated/laser irradiated samples relatively to controls was assessed by calculating the effect size (ES), adjusted for small sample numbers, and respective standard error, according to the equations provided in the literature [41]. The metabolic changes with absolute ES larger than 0.8 (and with standard error $<$ ES) were represented graphically and the two-sample $t$-test $(95 \%$ confidence level) was used to assess statistical significance of variations.

\subsection{Statistics}

Data are expressed as means \pm standard deviations of a representative of three experiments carried out in triplicate. Statistical analysis was performed using the Statistical Package for the Social Sciences (SPSS) version 19 software. Statistical comparisons were made by analysis of variance (ANOVA). Scheffé test was used for post hoc evaluations of differences among groups. In all of the statistical evaluations, $p<0.05$ was considered as statistically significant.

\section{Results and discussion}

\subsection{Synthesis and characterization of pegylated nGO sheets}

Nanographene oxide (nGO) sheets, successfully obtained by the modified Hummers' method, were characterized by AFM, XPS, zeta-potential $(\zeta)$ and DLS particle size analysis. The chemical exfoliation of graphite in aqueous media, followed by chemical activation and size separation by centrifugation, resulted in pegylated nGO sheets having an average thickness of around $9 \mathrm{~nm}$, corresponding to 13-14 layers (Fig. 1A). Moreover, these nGO sheets had a hydrodynamic size distribution in the $200-550 \mathrm{~nm}$ range, with the largest number of nanosheets presenting sizes around $300 \mathrm{~nm}$, as shown by DLS data (Fig. 1B). Pegylated nGO sheets were also characterized by XPS to study the elemental composition and chemical environment of the elements present within their surface. Fig. 1C shows a fitted $\mathrm{C} 1 \mathrm{~s}$ spectrum, composed by four components centered at: $284.8 \mathrm{eV}(\mathrm{C}-\mathrm{C} / \mathrm{C}=\mathrm{C}), 286.3 \mathrm{eV}(\mathrm{C}-\mathrm{O} / \mathrm{C}-$ $\mathrm{N}), 287.7 \mathrm{eV}(\mathrm{C}=\mathrm{O})$ and $288.9 \mathrm{eV}(\mathrm{O}-\mathrm{C}=\mathrm{O} / \mathrm{N}-\mathrm{C}=\mathrm{O})$. In general, the treatment carried out to promote the carboxylation of GO from surface hydroxyl and epoxide groups diminishes the intensity of the peak of the $\mathrm{C} 1 \mathrm{~s}$ spectrum associated to $\mathrm{C}-\mathrm{O}$ groups (at $286.3 \mathrm{eV}$ ). Thus, the high intensity of this peak after the pegylation process indicates successful incorporation of PEG-diamine. Finally, zeta-potential analysis was carried out to evaluate the surface charge of the nGO sheets. According to previously published data [38], the zeta-potential of chemically activated nGO sheets with $-\mathrm{COOH}$ surface groups is $-37.8 \pm 8 \mathrm{mV}$. After the pegylation process, the zeta-potential value changed slightly to $-32 \pm 6 \mathrm{mV}$, which confirms the existence of the positive amino ended branches.

\subsection{Cell uptake of pegylated nGO sheets and cell proliferation studies}

Uptake of the pegylated nGO sheets and its effects on the proliferation and viability of human osteosarcoma Saos-2 cells were 


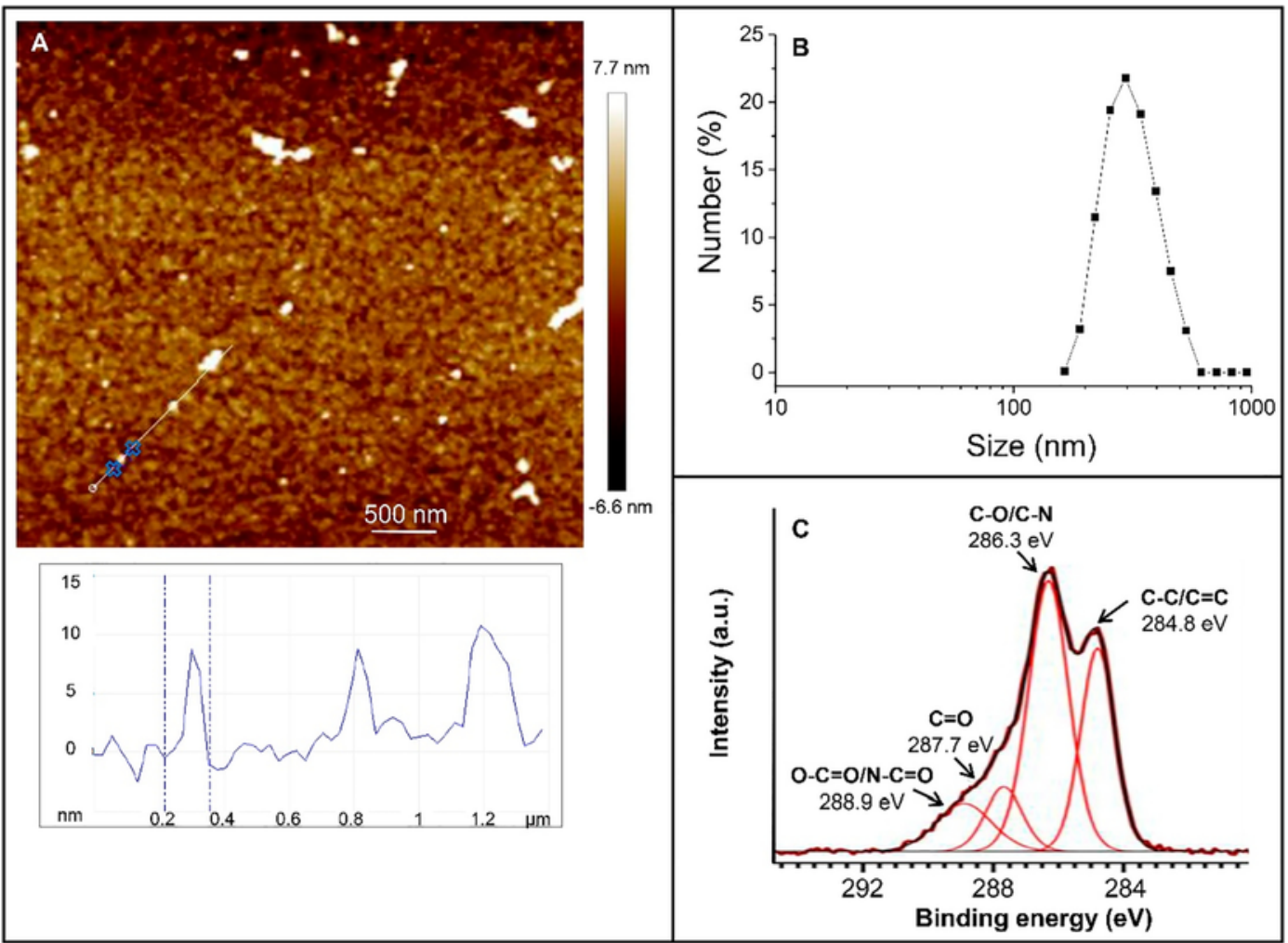

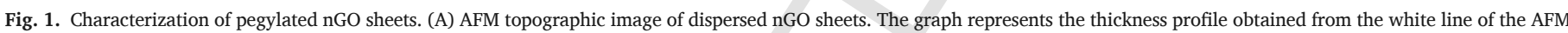
image. (B) nGO sheets particle size distribution obtained by DLS. (C) C1s XPS spectra obtained from nGO sheets.

evaluated after $24 \mathrm{~h}$ of incubation with $75 \mu \mathrm{g} / \mathrm{mL}$ of nGO. The internalization of pegylated nGO was confirmed by flow cytometry, after adding trypan blue to quench the fluorescence produced by the nGO adsorbed on the outer surface of cells (Fig. 2, orange fluorescence profile) [42].

As it can be observed, similar fluorescence profiles were obtained with and without trypan blue (orange and blue profiles, respectively), indicating that pegylated nGO sheets with $300 \mathrm{~nm}$ size

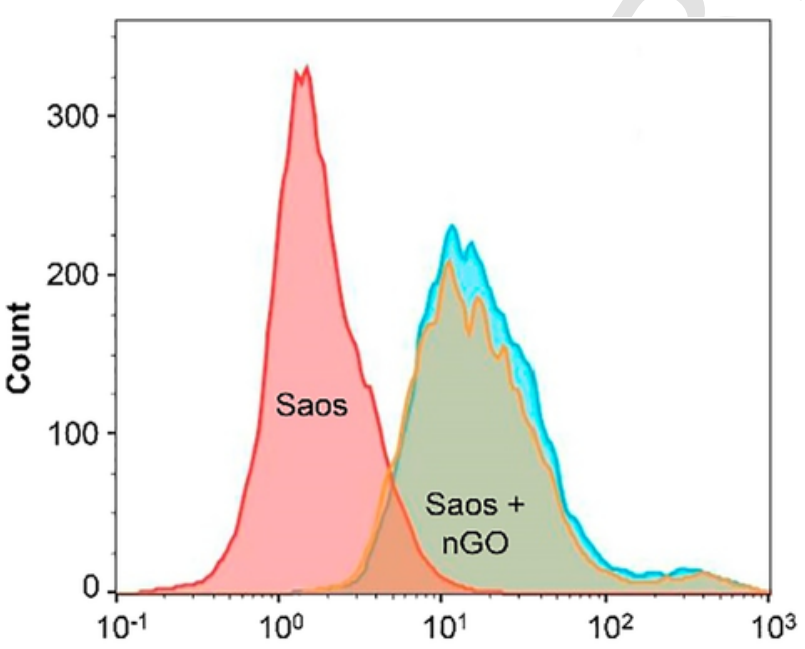

FL1 LOG:FITC LOG

Fig. 2. Cell uptake of pegylated nGO sheets by human Saos-2 cells: fluorescence profiles of Saos- 2 osteoblasts cultured for $24 \mathrm{~h}$ with $\mathrm{nGO}$ (Saos $+\mathrm{nGO}$ ) compared to control cells in absence of nanomaterial (Saos); the exterior cell surface fluorescence was quenched with trypan blue. (For interpretation of the references to color in this figure legend, the reader is referred to the web version of this article.) and a surface charge of $-32 \mathrm{mV}$ were completely internalized by human Saos-2 osteoblasts after $24 \mathrm{~h}$ of treatment. Confocal microscopy was additionally carried out to evaluate the Saos-2 morphology in the presence of pegylated nGO sheets and to study its intracellular localization. The results (Fig. S1) highlight that these pegylated nGO sheets have been internalized by Saos- 2 osteoblasts and were dispersed throughout the cytoplasm, preferentially localized in the perinuclear area, as indicated by arrows. The particles were clearly outside the nucleus and no signs of nuclear shrinkage (pyknosis) were apparent. These results are in agreement with those published by other authors, where most of the GO that entered cells distributed in the cytoplasm with very few particles being present in the nucleus $[43,44]$. Entrapment of nGO sheets in the lysosomes has also been reported [45,46]. For instance, Li and co-workers found that GO nanoassemblies were mainly distributed in acidic lysosomes of mouse embryonic fibroblasts (MEF) after their internalization [45]. Also, others have shown that, following lysosomal uptake, a small proportion of graphene could relocate to the cytosol and interact with the mitochondria $[47,48]$. The cytoskeleton is another key cellular component reported to interact with graphene-related nanomaterials. Matesanz and co-workers have shown GO nanosheets to localize on F-actin filaments of Saos-2 osteoblasts, and to affect cell cycle and death in a cytoskeleton-dependent manner [49]. Also, nGO was reported to cause disruption of F-actin cytoskeletal assembly within the lamellipodia of MDA-MB-231 breast cancer cells, thus impairing cell migration [47]. As for the uptake mechanism, although this was not assessed in the present study, it has been previously reported that Saos- 2 cells take up these pegylated nGO sheets mainly by macropinocytosis, although microtubule-dependent pathways may also be involved, without causing any morphological changes [50]. Moreover, Chartterjee and co-workers demonstrated that GO enters the cell 
through clathrin-mediated endocytosis as well as macropinocytosis [51].

The results obtained for viability and proliferation of human Saos-2 osteoblasts upon $24 \mathrm{~h}$ nGO treatment are shown in Fig. 3. High percentages of viability were obtained in all experimental conditions (97 and $93 \%$ in control and nGO-treated cells, respectively) and no significant differences were observed (Fig. 3A). With respect to the effect of nGO treatment on Saos proliferation (Fig. 3B), a significant delay in cell proliferation was observed, which is in agreement with previous results [49]. The high cell viability observed for nGO-exposed cells indicates that the plasma membrane integrity was highly preserved during the nano-bio interaction and subsequent nGO internalization by Saos- 2 cells. This is in contrast with other studies where graphene and graphene-based nanomaterials were shown to alter the dynamics and integrity of the plasma membrane during their internalization, inducing cell death [52]. Some authors suggested that the serious membrane disruption could be attributed to the strong electrostatic interactions between the graphene surface and the lipid bilayer of the cell membrane [53]. Others revealed that certain types of graphene, such as pristine graphene, could impair cell membrane integrity by regulation of membrane- and cytoskeleton-associated genes [54]. In the present study, the absence of membrane damage is likely due to surface functionalization of the nGO sheets by the non-toxic and non-immunogenic polymer PEG [55]. Indeed, this corroborates a previous study addressing the influence of nGO pegylation, whereby 1 - arm pegylated nGO showed high internalization and little interference with membrane integrity of Saos-2 and several other cell types [38].

As for the proliferation delay observed after nGO internalization (Fig. 3B), the results are in agreement with those published by other authors, where a cytotoxicity evaluation of graphene oxide on different cells types was carried out [56-58]. The possible mechanism involved in the Saos- 2 osteoblasts proliferation delay could be related to mitogen-activated protein kinases (MAPKs). It is well established that such kinases are involved in the regulation of cell growth, proliferation, migration and apoptosis [59]. Matesanz and co-workers reported that nGO sheets were localized on F-actin filaments of Saos-2 osteoblasts after their internalization, and thus altered cell cycle in a cytoskeleton-dependent manner [49]. Moreover, Tian and co-authors have recently published that nGO sheets retard cellular migration via disruption of actin cytoskeleton [60]. Therefore, considering that proliferation is dependent on the cell-cycle progress, it maybe suggested that the uptake of our graphene oxide nanosheets could induce cell-cycle alterations, which would cause the proliferation delay observed.

\subsection{Metabolic response to $n G O$ uptake and NIR laser irradiation}

The metabolic composition of human Saos-2 osteosarcoma cells was assessed by HRMAS ${ }^{1} \mathrm{H}$ NMR analysis of lysed cell pellets. A representative cells spectrum is shown in Fig. 4.
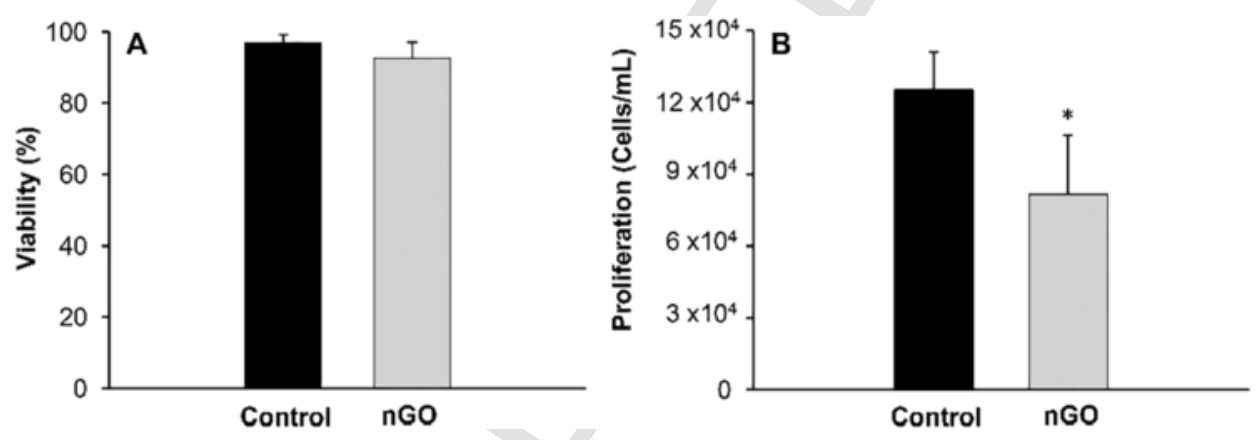

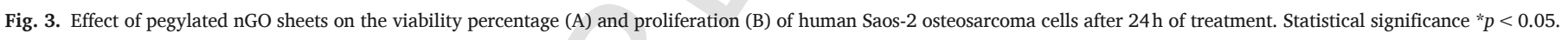

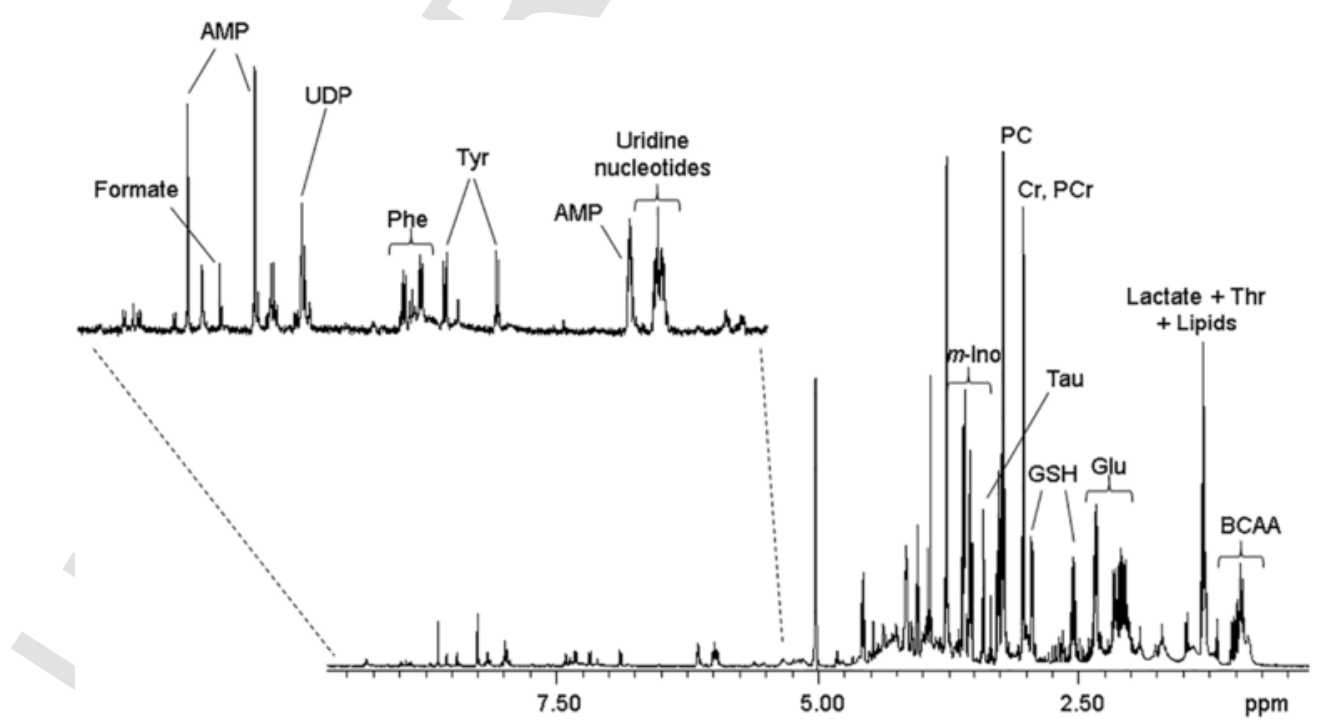

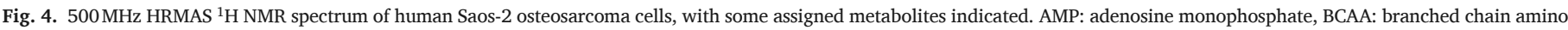

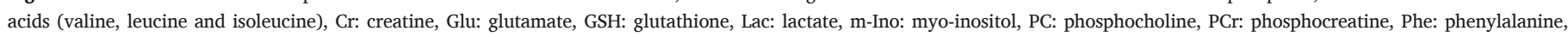
Tau: taurine, Thr: threonine, Tyr: tyrosine, UDP: uridine diphosphate. 
Similarly to what has been reported for other tumor cell lines [61-64], the signals of several amino acids (e.g., alanine, glutamate, glycine), reduced glutathione (GSH), lactate, creatine, choline-containing compounds, taurine, myo-inisitol and uridine/ adenosine nucleotides could be unambiguously identified. Phosphocholine was one of the most abundant metabolites detected, likely reflecting the high malignancy of the Saos-2 cell line, since this metabolite is considered a biomarker of tumor malignant transformation [65]. Other metabolites closely related to tumor progression, such as choline and creatine (reported to be inversely expressed in later stages of tumor growth), were also clearly detected. On the other hand, glucose was absent from the Saos-2 metabolic profile, in agreement with the composition reported for osteosarcoma [66] and other tumor cell lines [61]. In non-tumor cells, the glucose flux through the glycolytic pathway is regulated to maintain a constant concentration of adenosine triphosphate (ATP). By contrast, glucose uptake and glycolysis are known to occur nearly 10 times faster in most solid tumors compared with normal tissues [67], explaining the absence of this important metabolite in tumor cells.

To assess the impact of nGO uptake and laser irradiation on the metabolic profile of Saos-2 cells, multivariate analysis was applied to the spectra collected for the three sample groups, i.e. controls cells, nGO-exposed cells (nGO) and nGO-exposed and laser irradiated cells (nGO + laser). The resulting PCA scores scatter plot (Fig. 5A) showed that control and nGO-exposed cells were reasonably clustered and separated from each other (along the PC2 axis), while the laser-irradiated samples were more scattered, reflecting higher intra-group variability. Still, by applying PLS-DA, it was possible to discriminate the three sample groups along LV1 and LV2 axes (Fig. 5B) and to highlight the main metabolites accounting for such discrimination based on the corresponding loadings profiles (Fig. 5C and D). According to these profiles,

nGO samples differed from controls in the levels of uridine nucleotides, alanine, glutamine, glycine, taurine and creatine (Fig. 5C), while laser-irradiated samples were discriminated based on higher levels of glycerophosphocholine and AMP, together with lower levels of creatine and glutamate (Fig. 5D).

Spectral integration of individual metabolite signals was then carried out to assess the magnitude and statistical significance of the variations highlighted through multivariate analysis. The most relevant changes (absolute effect size $>0.8$, as justified in reference 41 ) are summarized in Fig. 6.

In comparison to control cells, Saos-2 cells exposed to nGO showed consistent alterations in 10 metabolites (first column of the heatmap shown in Fig. 6), namely decreases in taurine $(-44 \%)$, glutamine $(-35 \%)$, alanine $(-34 \%)$, creatine $(-33 \%)$, glycine $(-26 \%)$, methionine $(-25 \%)$ and glutamate $(-15 \%)$, together with increases in phosphocholine $(+24 \%)$, uridine nucleotides $(+44 \%)$ and AMP $(+59 \%)$. As for the effects of laser irradiation, compared to nGO-exposed cells, cells irradiated after nGO uptake showed higher levels of glycerophosphocholine $(+38 \%)$ and AMP $(+82 \%)$, together with decreased levels of glutamate $(-34 \%)$ and uridine nucleotides $(-20 \%)$ (second column of the heatmap shown in Fig. 6).

One of the main metabolic alterations displayed by Saos- 2 cells upon nGO uptake and subsequent laser irradiation was a cumulative increase in the levels of AMP, suggesting that cellular energetic homeostasis was affected. The impact of graphene on mitochondrial function and energy production has been previously reported for other cell types $[47,68,69]$. For instance, in liver HepG2 cells, nGO sheets $(400 \mathrm{~nm})$ were reported to cause dysregulation of mitochondrial $\mathrm{Ca}^{2+}$ homeostasis and a decrease in mitochondrial membrane potential (MMP), which is crucial for ATP synthesis [68]. In another work, pegylated nGO sheets in the 100-200 nm size range were shown to interact with the mitochondria of MDA-MB-231 breast tumor cells and to reduce ATP generation, possibly in relation with the down-regulation of enzymes involved in the TCA cycle and oxidative phosphorylation [47]. Moreover, in mouse alveolar macrophages, GO has been proposed to participate in redox reactions with components of the mitochondrial electron transport chain, thereby leading to impairment of ATP production [69]. In the present study, ATP was not clearly detected in the ${ }^{1} \mathrm{H}$ NMR spectra, possibly due its rapid turnover. Still, the observed accumulation of AMP could be an indirect indication of energy shortage, reflecting the attempt of adenylate kinase to rescue ATP from ADP, with the concomitant production of AMP. Indeed, increased ATP turnover and depletion of energy reservoirs have also been highlighted as hyperthermia-related effects due to increased metabolic rates [70]. In turn, a high level of AMP is recognized as an efficient activator of the AMP-activated protein kinase (AMPK), which is a crucial sensor of cellular energy status [71].

Upon activation, AMPK acts to restore energy homeostasis by stimulating catabolic reactions, while inhibiting ATP-consuming anabolic processes [71]. Interestingly, in the present study, the metabolic response to nGO comprised a consistent decrease in the levels of several amino acids, all of which could be used as anaplerotic substrates for the tricarboxylic acid (TCA) cycle, presumably to increase energy production. Upon laser irradiation, nGO-exposed cells further decreased their glutamate levels, while the other amino acids levels (alanine, glutamine, methionine) remained similar to non-irradiated nGO-exposed cells. Moreover, nGO-internalizing cells showed decreased levels of creatine (synthetized from arginine and glycine), which could also relate to the impact of $\mathrm{nGO}$ on amino acid metabolism. On the other hand, phosphocholine registered a relevant increase in nGO-internalizing cells in relation to controls. As phosphocholine is central to the synthesis of the major cell membrane component phosphatidylcholine, its increment may reflect the down-regulation of phospholipid synthesis, in consonance with the putative AMPK-mediated inhibition of anabolic processes. This also agrees with the proliferation delay displayed by nGO-exposed Saos-2 cells (Fig. 3B). Altogether, the metabolic variations described above suggest that AMPK activation may be an important player in the cellular responses to nGO and nGO-mediated hyperthermia, a hypothesis which needs to be verified in further studies.

Laser-irradiated cells further showed an increase in glycerophosphocholine, a well-known membrane breakdown product, likely reflecting heat-induced membrane damage [72]. Indeed, previous results demonstrated that nGO mediated hyperthermia under similar conditions to those applied in this study (low power and $7 \mathrm{~min}$ of laser exposure) caused Saos- 2 osteoblasts death by mixed apoptotic/necrotic process, implying loss of the plasma membrane integrity [30]. Notably, glycerophosphocholine levels did not vary upon nGO uptake in the absence of laser treatment, in agreement with the preservation of membrane integrity in non-irradiated nGO-internalizing cells (Fig. 3A).

As taurine is an antioxidant metabolite [73], its decrease upon nGO uptake could be related to nanomaterial-induced oxidative stress. However, while the generation of reactive oxygen species (ROS) has been reported to be a common response to graphene-based nanomaterials $[44,51]$, previous results from our group have shown that pegylated nGO sheets of similar size did not increase ROS in Saos-2 cells [49]. Moreover, glutathione, another important antioxidant metabolite clearly detected in the cells profile, did not vary upon nGO uptake, corroborating the absence of a strong oxidative stress response. Therefore, taurine variation likely relates to other possible roles of this metabolite, such as in osmoregulation, 

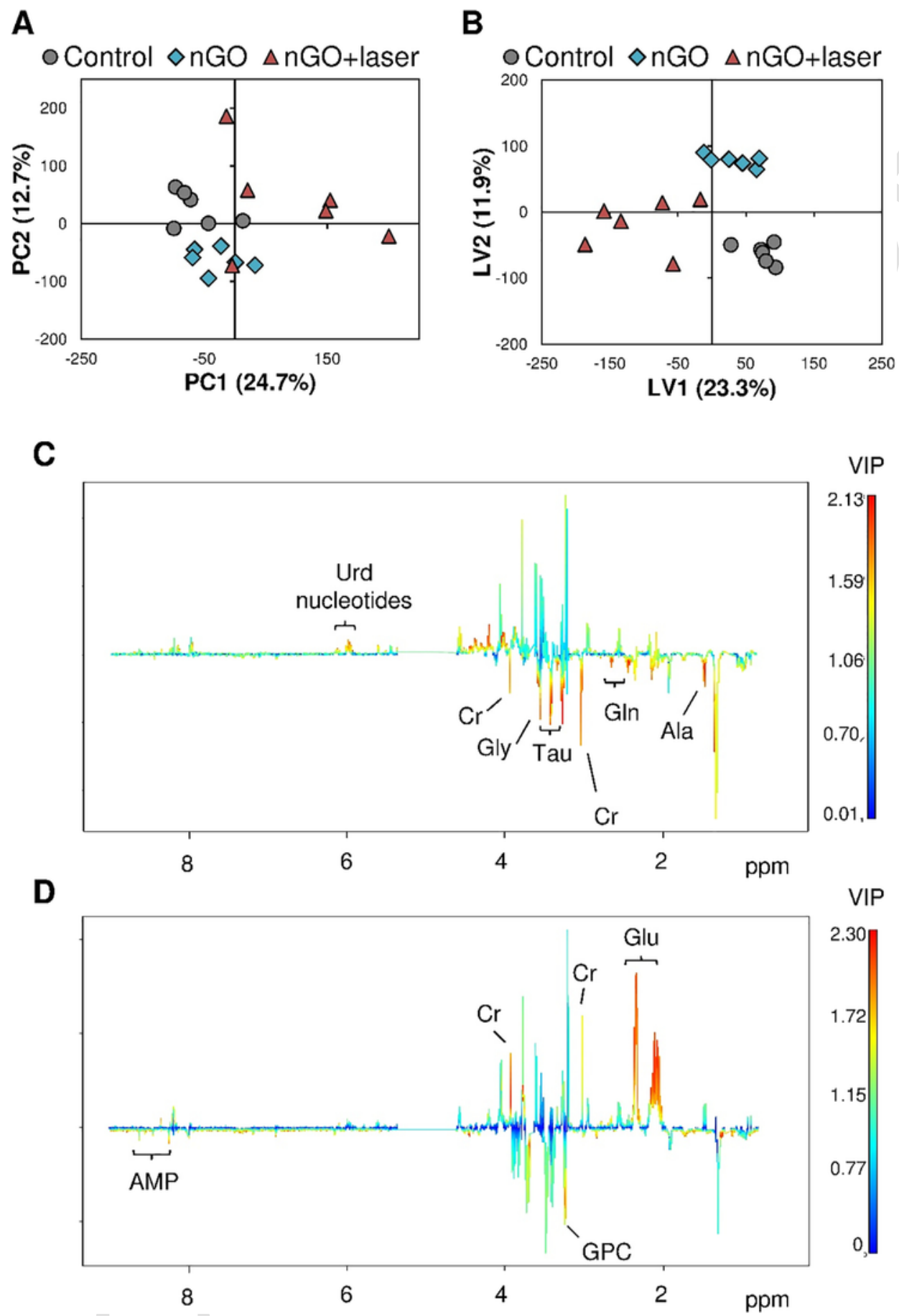

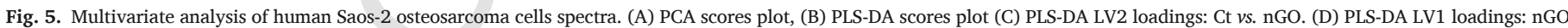
vs. $\mathrm{nGO}+$ laser.

membrane stabilization, calcium homeostasis or protein phosphorylation [74].

Finally, nGO-internalizing cells showed increased levels of uridine nucleotides, which subsequently decreased after laser irradiation. The bioavailability of uridine is particularly crucial to the synthesis of RNA and biomembranes (via the formation of pyrimidine nucleotide-lipid conjugates), being also needed for the formation of UDP-sugar conjugates involved in the post-translational modification of proteins [75]. Therefore, the observed variations may possibly relate to the effects of nGO and hyperthermia on cell proliferation and/or protein glycosylation.

\section{Conclusions}

Pegylated nGO sheets with a hydrodynamic size of $300 \mathrm{~nm}$ were taken up by human Saos-2 osteosarcoma cells without impairing cell viability, while causing a delay in cell proliferation. At the metabolic level, nGO produced consistent variations in several metabolites, mainly reflecting changes in energy homeostasis. In particular, based on increased levels of AMP and phosphocholine, together with decreases in several amino acids, we have newly hypothesized that AMPK activation may be an important player in 


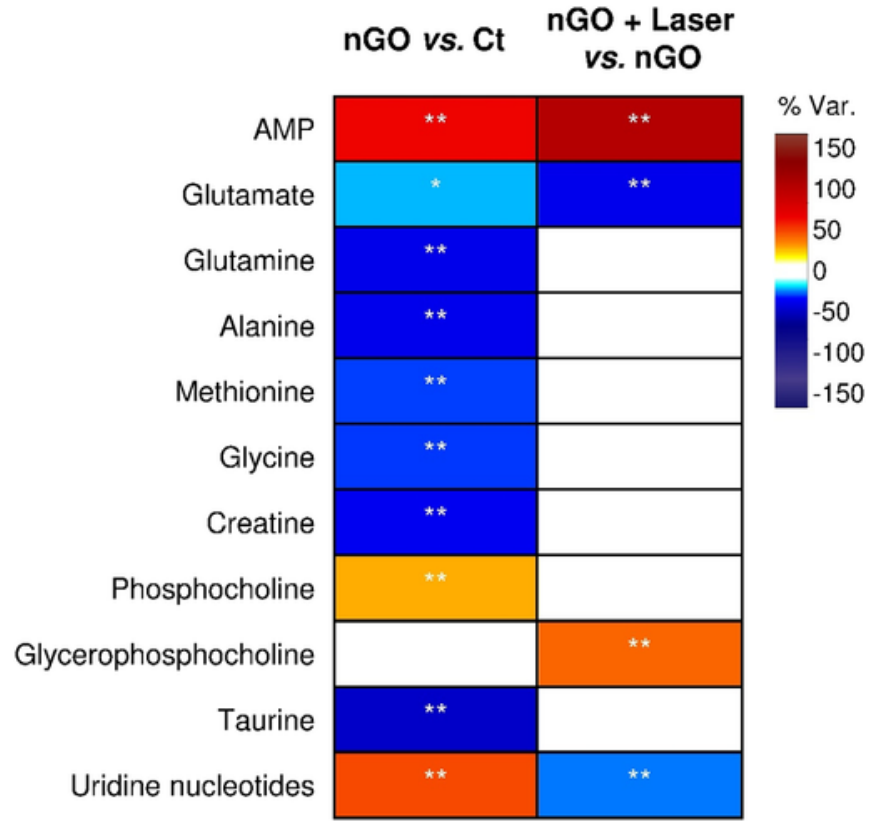

Fig. 6. Heatmap of main metabolic variations after nGO uptake by human Saos- 2 osteoblasts (nGO vs. Ct) and after NIR laser irradiation at $1.5 \mathrm{~W} / \mathrm{cm}^{2}$ power during $5 \mathrm{~min}$ (nGO + Laser $v s . \mathrm{nGO})$. Statistical significance $* * p<0.01$.

the cellular responses to nGO. Notably, subsequent laser irradiation of nGO-internalizing cells produced fewer effects: changes in AMP and glutamate were amplified, the levels of uridine nucleotides recovered and glycerophosphoholine increased, this latter change likely reflecting laser-induced membrane damage. Overall, this study has shown that cellular metabolome sensitively responded to nGO exposure and nGO-mediated hyperthermia, and that NMR metabolomics is a powerful tool in nanotoxicity studies, allowing for new hypotheses on molecular mechanisms underlying treatment responses to be generated. Furthermore, knowledge on the thermosensitivity of metabolite changes may be important to modulate cell killing by hyperthermia in future works.

Supplementary data to this article can be found online at https:// doi.org/10.1016/j.msec.2018.05.057.

\section{Acknowledgements}

This work was developed within the scope of the project CICECO-Aveiro Institute of Materials, POCI-01-0145-FEDER-007679 (FCT Ref. UID/CTM/50011/2013), financed by national funds through the FCT/MEC and when appropriate co-financed by FEDER under the PT2020 Partnership Agreement. M.C. acknowledges the FCT financial support [Post-Doctoral Grant SFRH/BPD/101468/2014] and Operational Program Human Capital (POCH), European Union. H.O. acknowledges financial support FCT SFRH/BPD/111736/2015. M.T.P. acknowledges funding from Ministerio de Economía y Competitividad (projects MAT2013-43299-R and MAT2016-75611-R AEI/FEDER, UE). M.V.R. acknowledges funding from the European Research Council (Advanced Grant VERDI; ERC-2015-AdG Proposal No. 694160). I.F.D. acknowledges the FCT/MCTES for a research contract under the Program "Investigador FCT" 2014. The authors further acknowledge the financial support from the European Union Framework Programme for Research and Innovation HORIZON 2020, under the TEAMING Grant agreement No 739572 - The Discoveries CTR. The Portuguese National NMR Network supported with FCT funds and Bruker BioSpin
$\mathrm{GmbH}$ for database access are also acknowledged. Thanks also to the staff of the Centro de Citometría y Microscopía de Fluorescencia of the Universidad Complutense de Madrid (Spain) and ICTS Centro Nacional de Microscopia Electrónica (Spain) for the assistance in confocal microscopy and AFM studies, respectively.

\section{References}

[1] B. Sumer, J. Gao, Theranostic nanomedicine for cancer, Nanomedicine 3 (2008) 137-140.

[2] L. Li, F. Tang, H. Liu, T. Liu, N. Hao, D. Chen, X. Teng, J. He, In vivo delivery of silica nanorattle encapsulated docetaxel for liver cancer therapy with low toxicity and high efficacy, ACS Nano 4 (2010) 6874-6882.

[3] Z. Zhang, L. Wang, J. Wang, X. Jiang, X. Li, Z. Hu, Y. Ji, X. Wu, C. Chen, Mesoporous silica-coated gold nanorods as a light-mediated multifunctional theranostic platform for cancer treatment, Adv. Mater. 24 (2012) 1418-1423.

[4] Y. Liu, K. Ai, J. Liu, M. Deng, Y. He, L. Lu, Dopamine-melanin colloidal nanospheres: an efficient near-infrared photothermal therapeutic agent for in vivo cancer therapy, Adv. Mater. 25 (2013) 1353-1359.

[5] L. Cheng, C. Wang, L. Feng, K. Yang, Z. Liu, Functional nanomaterials for phototherapies of cancer, Chem. Rev. 114 (2014) 10869-10939.

[6] Yang Yu, L. Jingjing, S. Xiaoqi, F. Liangzhu, Z. Wenwen, L. Zhuang, C. Meiwan, Near-infrared light-activated cancer cell targeting and drug delivery with aptamer-modified nanostructures, Nano Res. 9 (2016) 139-148.

[7] J. Beik, Z. Abed, F.S. Ghoreishi, S. Hosseini-Nami, S. Mehrzadi, A. Shakeri-Zadeh, S.K. Kamrava, Nanotechnology in hyperthermia cancer therapy: from fundamental principles to advanced applications, J. Control. Release 235 (2016) 205-221.

[8] G. Fiorentini, A. Szasz, Hyperthermia today: electric energy, a new opportunity in cancer treatment, J. Cancer Res. Ther. 2 (2006) 41-46.

[9] B. Van de Broek, N. Devoogdt, A. D'Hollander, H.L. Gijs, K. Jans, L. Lagae, S Muyldermans, G. Maes, G. Borghs, Specific cell targeting with nanobody conjugated branched gold nanoparticles for photothermal therapy, ACS Nano 5 (2011) 4319-4328.

[10] L. Li-Sen, C. Zhong-Xiao, C. Jian-Bo, K. Kai-Mei, P. Qiao-Li, G. Jinhao, Y. Huang-Hao, L. Gang, C. Xiaoyuan, Multifunctional $\mathrm{Fe}_{3} \mathrm{O}_{4} @$ polydopamine core-shell nanocomposites for intracellular mRNA detection and imaging-guided photothermal therapy, ACS Nano 8 (2014) 3876-3883.

[11] S. Kossatz, J. Grandke, P. Couleaud, A. Latorre, A. Aire, K. Crosbie-Staunton, R. Ludwig, H. Dähring, V. Ettelt, A. Lazaro-Carrillo, M. Calero, M. Sader, J. Courty, Y. Volkov, A. Prina-Mello, A. Villanueva, Á. Somoza, A.L. Cortajarena, R. Miranda, I. Hilger, Efficient treatment of breast cancer xenografts with multifunctionalized iron oxide nanoparticles combining magnetic hyperthermia and anti-cancer drug delivery, Breast Cancer Res. 17 (2015) 1-17.

[12] Z. Zhang, J. Wang, C. Chen, Near infrared light mediated nanoplatforms for cancer thermo-chemotherapy and optical imaging, Adv. Mater. 25 (2013) 3869-3880.

[13] J. Zhou, Z. Lu, X. Zhu, X. Wang, Y. Liao, Z. Ma, F. Li, NIR photothermal therapy using polyaniline nanoparticles, Biomaterials 34 (2013) 9584-9592.

[14] K. Yang, et al., In vitro and in vivo near infrared photothermal therapy of cancer using polypyrrole organic nanoparticles, Adv. Mater. 24 (2012) 5586-5592.

[15] J. Chen, C. Glaus, R. Laforest, Q. Zhang, M. Yang, M. Gidding, M.J. Welch, Y. Xia, Gold nanocages as photothermal transducers for cancer treatment, Small 6 (2010) 811-817.

[16] L. Huiyu, C. Dong, L. Linlin, L. Tianlong, T. Longfei, W. Xiaoli, T. Fanggiong, Multifunctional gold nanoshells on silica nanorattles: a platform for the combination of photothermal therapy and chemotherapy with low systemic toxicity, Angew. Chem. 123 (2011) 921-925.

[17] Z. Zha, X. Yue, Q. Ren, Z. Dai, Uniform polypyrrole nanoparticles with high photothermal conversion efficiency for photothermal ablation of cancer cells, Adv. Mater. 25 (2013) 777-782.

[18] Y. Li, W. Lu, Q. Huang, C. Li, W. Chen, Copper sulfide nanoparticles for photothermal ablation of tumor cells, Nanomedicine 5 (2010) 1161-1171.

[19] S.S. Chou, B. Kaehr, J. Kim, B.M. Foley, M. De, P.E. Hopkins, J. Huang, C.J. Brinker, V.P. Dravid, Chemically exfoliated $\mathrm{MoS}_{2}$ as near-infrared photothermal agents, Angew. Chem. 125 (2013) 4254-4258.

[20] L. Qin, S. Chunyang, H. Qun, L. Daobin, K. Adnan, X. Ting, W. Ziyu, W. Jun, S. Li, Ultrathin carbon layer coated $\mathrm{MoO}_{2}$ nanoparticles for high-performance near-infrared photothermal cancer therapy, Chem. Commun. 51 (2015) 10054-10057.

[21] B. Tian, C. Wang, S. Zhang, L. Feng, Z. Liu, Photothermally enhanced photodynamic therapy delivered by nano-graphene oxide, ACS Nano 5 (2011) 7000-7009. 
[22] W. Miao, G. Shim, S. Lee, Y.S. Choe, Y.K. Oh, Safety and tumor tissue accumulation of pegylated graphene oxide nanosheets for co-delivery of anticancer drug and photosensitizer, Biomaterials 34 (2013) 3402-3410.

[23] Z. Sheng, L. Song, J. Zheng, D. Hu, M. He, M. Zheng, G. Gao, P. Gong, P. Zhang, Y. Ma, L. Cai, Protein-assisted fabrication of nano-reduced graphene oxide for combined in vivo photoacoustic imaging and photothermal therapy, Biomaterials 34 (2013) 5236-5243.

[24] Z. Yanwu, M. Shanthi, C. Weiwei, L. Xuesong, S. Ji Won, R.P. Jeffrey, S.R. Rodney, Graphene and graphene oxide: synthesis, properties, and applications, Adv. Mater. 22 (2010) 3906-3924.

[25] J.T. Robinson, S.M. Tabakman, Y. Liang, H. Wang, H. Sanchez Casalongue, D. Vinh, H. Dai, Ultrasmall reduced graphene oxide with high near-infrared absorbance for photothermal therapy, J. Am. Chem. Soc. 133 (2011) 6825-6831.

[26] Y. Wang, H. Wang, D. Liu, S. Song, X. Wang, H. Zhang, Graphene oxide covalently grafted upconversion nanoparticles for combined NIR mediated imaging and photothermal/photodynamic cancer therapy, Biomaterials 34 (2013) 7715-7724.

[27] Z.M. Markovic, L.M. Harhaji-Trajkovic, B.M. Todorovic-Markovic, D.P. Kepić, K.M. Arsikin, S.P. Jovanović, A.C. Pantovic, M.D. Dramićanin, V.S. Trajkovic, In vitro comparison of the photothermal anticancer activity of graphene nanoparticles and carbon nanotubes, Biomaterials 32 (2011) 1121-1129.

[28] H. Zhang, H. Wu, J. Wang, Y. Yang, D. Wu, Y. Zhang, Y. Zhang, Z. Zhou, S. Yang, Graphene oxide-BaGdF 5 nanocomposites for multi-modal imaging and photothermal therapy, Biomaterials 42 (2015) 66-77.

[29] C. Jianbo, A. Hengqing, H. Xinglu, F. Guifeng, Z. Rongqiang, Z. Lei, Jin Xie, Fan Zhang, Monitoring of the tumor response to nano-graphene oxide-mediated photothermal/photodynamic therapy by diffusion-weighted and BOLD MRI, Nano 8 (2016), 10152.

[30] M. Vila, M.C. Matesanz, G. Gonçalves, M.J. Feito, J. Linares, P.A.A.P. Marques, M.T. Portolés, M. Vallet-Regí, Triggering cell death by nanographene oxide mediated hyperthermia, Nanotechnology 035101 (7pp) (2014) 25.

[31] W. Shim, M.J. Paik, D.T. Nguyen, J.K. Lee, Y. Lee, J.H. Kim, E.H. Shin, J.S. Kang, H.S. Jung, S. Choi, S. Park, J.S. Shim, G. Lee, Analysis of changes in gene expression and metabolic profilesinduced by silica-coated magnetic nanoparticles, ACS Nano 6 (2012) 7665-7680.

[32] H. Xiangang, O. Shaohu, M. Li, A. Jing, Z. Qixing, Effects of gsraphene oxide and oxidized carbon nanotubes on the cellular division, microstructure, uptake, oxidative stress, and metabolic profiles, Environ. Sci. Technol. 49 (2015) 10825-10833.

[33] C.K. Larive, G.A. Barding, M.M. Dinges, NMR spectroscopy for metabolomics and metabolic profiling, Anal. Chem. 87 (2015) 133-146.

[34] S.B. Rodan, Y. Imai, M.A. Thiede, G. Wesolowski, D. Thompson, Z. Bar-Shavit, S. Shull, K. Mann, G.A. Rodan, Characterization of a human osteosarcoma cell line (Saos-2) with osteoblastic properties, Cancer Res. 47 (1987) 4961-4966.

[35] L. Fassina, L. Visai, L. Asti, F. Benazzo, P. Speziale, M.C. Tanzi, G. Magenes, Calcified matrix production by SAOS-2 cells inside a polyurethane porous scaffold, using a perfusion bioreactor, Tissue Eng. 11 (2005) 685-700.

[36] U. Mayr-Wohlfart, J. Fiedler, K.P. Günther, W. Puhl, S. Kessler, Proliferation and differentiation rates of a human osteoblast-like cell line (SaOS-2) in contact with different bone substitute materials, J. Biomed. Mater. Res. A 57 (2001) 132-139.

[37] G. Gonçalves, P.A.A.P. Marques, C. Granadeiro, H.I.S. Nogueira, M.K. Singh, J. Grácio, Surface modification of graphene nanosheets with goldnanoparticles: the role of oxygen moieties at graphenesurface on gold nucleation and growth, Chem. Mater. 21 (2009) 4796-4802.

[38] M. Vila, M.T. Portolés, P.A.A.P. Marques, M.J. Feito, M.C. Matesanz, C. Ramírez-Santillán, G. Gonçalces, S.M.A. Cruz, A. Nieto, M. Vallet-Regí, Cell uptake survey of pegylated nanographene oxide, Nanotechnology 465103 (9pp) (2012) 23.

[39] I.F. Duarte, J. Marques, A.F. Ladeirinha, C.M. Rocha, I. Lamego, R. Calheiros, T.M. Silva, M.P.M. Marques, J.B. Melo, I.M. Carreira, A.M. Gil, Analytical approaches towards successful human cell metabolome studies by NMR spectroscopy, Anal. Chem. 81 (2009) 5023-5032.

[40] D.S. Wishart, T. Jewison, A.C. Guo, M. Wilson, C. Knox, Y. Liu, Y. Djoumbou, R. Mandal, F. Aziat, E. Dong, S. Bouatra, I. Sinelnikov, D. Arndt, J. Xia, P. Liu, F. Yallou, T. Bjorndahl, R. Perez-Pineiro, R. Eisner, F. Allen, V. Neveu, R. Greiner, A. Scalbert, HMDB 3.0 - The human metabolome database in 2013, Nucleic Acids Res. 41 (2013) D801-D807.

[41] L. Berben, S.M. Sereika, S. Engberg, Effect size estimation: methods and examples, Int. J. Nurs. Stud. 49 (2012) 1039-1047.

[42] W. Busch, S. Bastian, U. Trahorsch, M. Iwe, D. Kühnel, T. Meißner, A. Springer, M Gelinsky, V. Richter, C. Ikonomidou, A. Potthoff, I. Lehmann, K. Schirmer, Internalisation of engineered nanoparticles into mammalian cells in vitro: influence of cell type and particle properties, J. Nanopart. Res. 13 (2010) 293-310.

[43] K. Wang, J. Ruan, H. Song, J. Zhang, Y. Wo, S. Guo, D. Cui, Biocompatibility of graphene oxide, Nanoscale Res. Lett. 6 (2010) 8.
[44] B. Zhang, P. Wei, Z. Zhou, T. Wei, Interactions of graphene with mammalian cells: molecular mechanisms and biomedical insights, Adv. Drug Deliv. Rev. 105 (2016) $145-162$.

[45] Y.Y. Li, Z.Z. Lu, Z.J. Li, G.J. Nie, Y. Fang, Cellular uptake and distribution of graphene oxide coated with layer-by-layer assembled polyelectrolytes, J. Nanopart. Res. 16 (2014) 2384.

[46] Y.P. Zeng, Z.Y. Yang, S.L. Luo, H. Li, C. Liu, Y.H. Hao, J. Liu, W.D. Wang, R. Li, Fast and facile preparation of PEGylated graphene from graphene oxide by lysosome targeting delivery of photosensitizer to efficiently enhance photodynamic therapy, RSC Adv. 5 (2015) 57725-57734.

[47] H. Zhou, B. Zhang, J. Zheng, M. Yu, T. Zhou, K. Zhao, Y. Jia, X. Gao, C. Chen, T. Wei, The inhibition of migration and invasion of cancer cells by graphene via the impairment of mitochondrial respiration, Biomaterials 35 (2014) 1597-1607.

[48] Y.P. Li, Q.L. Wu, Y.L. Zhao, Y.F. Bai, P.S. Chen, T. Xia, D.Y. Wang, Response of microRNAs to in vitro treatment with graphene oxide, ACS Nano 8 (2014) 2100-2110.

[49] M.C. Matesanz, M. Vila, M.J. Feito, J. Linares, G. Gonçalves, M. Vallet-Regí, P.A.A.P. Marques, M.T. Portolés, The effects of graphene oxide nanosheets localized on F-actin filaments oncell-cycle alterations, Biomaterials 34 (2013) 1562-1569.

[50] J. Linares, M.C. Matesanz, M. Vila, M.J. Feito, G. Gonçalves, M. Vallet-Regí, P.A.A.P. Marques, M.T. Portolés, Endocytic mechanisms of graphene oxide nanosheets in osteoblasts, hepatocytes and macrophages, ACS Appl. Mater. Interfaces 6 (2014) 13697-13706.

[51] N. Chatterjee, H.-J. Eom, J. Choi, A systems toxicology approach to the surface functionality control of graphene-cell interactions, Biomaterials 35 (2014) 1109-1127.

[52] S. Syama, P.V. Mohanan, Safety and biocompatibility of graphene: a new generation nanomaterial for biomedical application, Int. J. Biol. Macromol. 86 (2016) 546-555.

[53] K.H. Liao, Y.S. Lin, C.W. Macosko, C.L. Haynes, Cytotoxicity of graphene oxide and graphene in human erythrocytes and skin fibroblasts, ACS Appl. Mater. Interfaces 3 (2011) 2607-2615.

[54] M. Xu, J. Zhu, F. Wang, Y. Xiong, Y. Wu, Q. Wang, J. Weng, Z. Zhang, W. Chen, S. Liu, Improved in vitro and in vivo biocompatibility of graphene oxide through surface modification: poly(acrylic acid)-functionalization is superior to PEGylation, ACS Nano 10 (2016) 3267-3281.

[55] R. Shi, Polyethylene glycol repairs membrane damage and enhances functional recovery: a tissue engineering approach to spinal cord injury, Neurosci. Bull. 29 (2013) 460-466.

[56] Y. Talukdar, J.T. Rashkow, G. Lalwani, S. Kanakia, B. Sitharaman, The effects of graphene nanostructures on mesenchymal stem cells, Biomaterials 35 (2014) 4863-4877.

[57] L. Horváth, A. Magrez, M. Burghard, K. Kern, L. Forró, B. Schwaller, Evaluation of the toxicity of graphene derivatives on cells of the lung luminal surface, Carbon 64 (2013) 45-60.

[58] Y. Chang, S.T. Yang, J.H. Liu, E. Dong, Y. Wang, A. Cao, Y. Liu, H. Wang, In vitro toxicity evaluation of graphene oxide on A549 cells, Toxicol. Lett. 200 (2011) 201-210.

[59] T. Boutros, E. Chevet, P. Metrakos, Mitogen-activated protein (MAP) kinase/MAP kinase phosphatase regulation: roles in cell growth, death, and cancer, Pharmacol. Rev. 60 (2008) 261-310.

[60] X. Tian, Z. Yang, G. Duan, A. Wu, Z. Gu, L. Zhang, C. Chen, Z. Chai, C. Ge, R. Zhou, Graphene oxide nanosheets retard cellular migration via disruption of actin cytoskeleton, Small 13 (2017), 1602133.

[61] R.M. Maria, W.F. Altei, A.D. Andricopulo, A.B. Becceneri, M.R. Cominetti, T. Venâncio, L.A. Colnago, Characterization of metabolic profile of intact non-tumor and tumor breast cells by high-resolution magic angle spinning nuclear magnetic resonance spectroscopy, Anal. Biochem. 488 (2015) 14-18.

[62] T.A. Fedele, A.C. Galdos-Riveros, H.J. de Farias e Melo, A. Magalhães, A.M. Durvanei, Prognostic relationship of metabolic profile obtained of melanoma B16F10, Biomed Pharmacother 67 (2013) 146-156.

[63] M. Bayet-Robert, D. Loiseau, P. Rio, A. Demidem, C. Barthomeuf, G. Stepien, D. Morvan, Quantitative two-dimensional HRMAS 1H-NMR spectroscopy-based metabolite profiling of human cancer cell lines and response to chemotherapy, Magn. Reson. Med. 63 (2010) 1172-1183.

[64] A.F. da Silva, D. Sartori, F.C. Macedo, L.R. Ribeiro, M.H.P. Fungaro, M.S. Mantovani, Effects of b-glucan extracted fromAgaricus blazei on the expressionof ERCC5, CASP9, and CYP1A1 genesand metabolic profile in HepG2 cells, Hum. Exp. Toxicol 32 (2013) 647-654.

[65] G. Eliyahu, T. Kreizman, H. Degani, Phosphocholine as a biomarker of breast cancer: molecular and biochemical studies, Int. J. Cancer 120 (2007) 1721-1730.

[66] I.F. Duarte, I. Lamego, J. Marques, M.P. Marques, B.J. Blaise, A.M. Gil, Nuclear magnetic resonance (NMR) study of the effect of cisplatin on the metabolic profile of MG-63 osteosarcoma cells, J. Proteome Res. 9 (2010) 5877-5886.

[67] D.L. Nelson, M.M. Cox, Lehninger Principles of Biochemistry, W. H. Freeman, San Francisco, 2008

[68] T. Lammel, P. Boisseaux, M.L. Fernandez-Cruz, J.M. Navas, Internalization and cytotoxicity of graphene oxide and carboxyl graphene nanoplatelets in the human hepatocellular carcinoma cell line Hep G2, Part. Fibre Toxicol. 10 (2013) 21pp. 
[69] M.C. Duch, G.R.S. Budinger, Y.T. Liang, S. Soberanes, D. Urich, S.E. Chiarella, L.A. Campochiaro, A. Gonzalez, N.S. Chandel, M.C. Hersam, G.M. Mutlu, Minimizing oxidation and stable nanoscale dispersion improves the biocompatibility of graphene in the lung, Nano Lett. 11 (2011) 5201-5207.

[70] C. Streffer, Metabolic changes during and after hyperthermia, Int. J. Hyprthermia 1 (1985) 305-319.

[71] C.A. Peixoto, W.H. de Oliveira, S.M. da Racho Araújo, A.K. Santana Nunes, AMPK activation: role in the signaling pathways of neuroinflammation and neurodegeneration, Exp. Neurol. 298 (2017) 31-41.

[72] M. Milkevitch, H. Shim, U. Pilatus, S. Pickup, J.P. Wehrle, D. Samid, H. Poptani, J.D. Glickson, E.J. Delikatny, Increases in NMR-visible lipid and glycerophosphocholine during phenylbutyrate-induced apoptosis in human prostate cancer cells, Biochim. Biophys. Acta, Mol. Cell Biol. Lipids 1734 (2005) 1-12.

[73] C.J. Jong, J. Azuma, S. Schaffer, Mechanism underlying the antioxidantactivity of taurine: prevention of mitochondrial oxidant production, Amino Acids 42 (2012) 2223-2232.

[74] S.W. Schaffer, C.J. Jong, R. KC, J. Azuma, Physiological roles of taurine in heart and muscle, J. Biomed. Sci. 17 (Suppl. 1) (2010) S2.

[75] G.P. Connolly, J.A. Duley, Uridine and its nucleotides: biological actions, therapeutic potentials, Trends Pharmacol. Sci. 20 (1999) 218-225. 
As it can be observed, similar fluorescence profiles were obtained with and without trypan blue (orange and blue profiles, respectively), indicating that pegylated nGO sheets with $300 \mathrm{~nm}$ size and a surface charge of $-32 \mathrm{mV}$ were completely internalized by human Saos-2 osteoblasts after $24 \mathrm{~h}$ of treatment. Confocal microscopy was additionally carried out to evaluate the Saos- 2 morphology in the presence of pegylated nGO sheets and to study its intracellular localization. The results (Fig. Fig. S1) highlight that these pegylated nGO sheets have been internalized by Saos-2 osteoblasts and were dispersed throughout the cytoplasm, preferentially localized in the perinuclear area, as indicated by arrows. The particles were clearly outside the nucleus and no signs of nuclear shrinkage (pyknosis) were apparent. These results are in agreement with those published by other authors, where most of the GO that entered cells distributed in the cytoplasm with very few particles being present in the nucleus $[43,44]$. Entrapment of nGO sheets in the lysosomes has also been reported $[45,46]$. For instance, $\mathrm{Li}$ and co-workers found that GO nanoassemblies were mainly distrib- uted in acidic lysosomes of mouse embryonic fibroblasts (MEF) after their internalization [45]. Also, others have shown that, following lysosomal uptake, a small proportion of graphene could relocate to the cytosol and interact with the mitochondria $[47,48]$. The cytoskeleton is another key cellular component reported to interact with graphene-related nanomaterials. Matesanz and co-workers have shown GO nanosheets to localize on F-actin filaments of Saos-2 osteoblasts, and to affect cell cycle and death in a cytoskeleton-dependent manner [49]. Also, nGO was reported to cause disruption of F-actin cytoskeletal assembly within the lamellipodia of MDA-MB-231 breast cancer cells, thus impairing cell migration [47]. As for the uptake mechanism, although this was not assessed in the present study, it has been previously reported that Saos- 2 cells take up these pegylated nGO sheets mainly by macropinocytosis, although microtubule-dependent pathways may also be involved, without causing any morphological changes [50]. Moreover, Chartterjee and co-workers demonstrated that GO enters the cell through clathrin-mediated endocytosis as well as macropinocytosis [51]. 